\title{
Intraoperative monitoring during surgery for acoustic neuroma: benefits of an extratympanic intrameatal electrode
}

\author{
N Mullatti, H B Coakham, A R Maw, S R Butler, M H Morgan
}

Department of Clinical Neurophysiology

N Mullatti

M H Morgan

Department of Neurosurgery, Frenchay Hospital, Bristol BS16 1LE, UK H B Coakham

Department of Otolaryngology, Bristol Royal Infirmary, Bristol BS2 8HW, UK

R Maw

Burden Neurological Institute, Stoke Lane, Stapleton, Bristol BS16 1QT, UK

S R Butler

Correspondence to: Dr N Mullatti, Department of Clinical Neurophysiology, Mapother House, King's College Hospital, De Crespigny Park, London SE5 9RS, UK

Received 13 August 1998 and in revised form 20 November 1998

Accepted 23 November 1998

\begin{abstract}
Objectives-To assess the utility of an extratympanic intrameatal electrode for intraoperative monitoring during acoustic neuroma and other cerebellopontine angle tumour surgery and to define the neurophysiological and surgical factors which influence hearing preservation.

Methods-Twenty two patients, 18 with acoustic neuromas and four with other cerebellopontine angle tumours, underwent intraoperative monitoring during tumour excision. The extratympanic intrameatal electrode (IME) was used to record the electrocochleogram (ECoG) and surface electrodes to record the brainstem auditory evoked response (ABR).

Results-The compound action potential (CAP) of the ECoG was two and a half times greater in amplitude than wave $I$ of the ABR and was easily monitored. Virtually instant information was available as minimal averaging was required. Continuous monitoring was possible from the commencement of anaesthesia to skin closure. The IME was easy to place, non-invasive, and did not interfere with the operative field. Operative procedures which affected CAP or wave $V$ latency or amplitude were drilling around the internal auditory meatus, tumour dissection, nerve section, and brainstem and cerebellar retraction. Hearing was achieved in $59 \%$ of patients.

Conclusions-The IME had significant benefits in comparison with other methods of monitoring. The technique provided information beneficial to preservation of hearing.
\end{abstract}

(f Neurol Neurosurg Psychiatry 1999;66:591-599)

Keywords: intraoperative monitoring; extratympanic intrameatal electrode; acoustic neuroma surgery; hearing preservation

Preservation of hearing has become an important issue in surgery for acoustic neuroma (vestibular schwannoma). ${ }^{1-4}$ With the aid of MRI, acoustic neuromas are now increasingly diagnosed before useful hearing is lost. Therefore, surgical procedures may be modified to preserve residual hearing. Intraoperative monitoring of the acoustic nerve has been shown to be a useful aid to hearing preservation by warning the surgeon of operative events which threaten the integrity of the acoustic nerve. ${ }^{235}$
Various methods of monitoring have been described in the literature. Initial reports described the use of intraoperative brainstem auditory evoked responses (ABRs) recorded with surface electrodes. ${ }^{5-7}$ This procedure was soon shown to have limitations. The degree of hearing impairment already present at the time of diagnosis meant that the ABR waveforms were often ill defined, of very low amplitude, and difficult to monitor. The need to average many responses inevitably introduced a critical time delay before the surgeon could be informed. Any gradual changes in latency were often lost in the process of averaging. In addition, it was found that the ABR was sensitive to numerous factors which were not necessarily related to relevant functional changes in the auditory pathways. These included systemic factors such as hypothermia or hypocarbia. ${ }^{8}$

In an attempt to overcome some of these problems Moller et al tried direct recording from the VIIIth nerve. ${ }^{9}$ The potentials, being of higher amplitude than the ABRs, required fewer stimuli to produce detectable results and thus reduced the delay before information could be conveyed to the surgeon. However, the major disadvantage of this method was the fact that monitoring could only be performed once the VIIIth nerve had been exposed, and inevitably the electrode placement was likely to interfere with the operative field. ${ }^{10}{ }^{11}$

An alternative method of recording is by means of transtympanic electrodes resting on the promontory, which record electrocochleograms (ECoGs) ${ }^{12}{ }^{13}$ This did not have the above disadvantages. The recording electrode position is nearer the generator site of the cochlear nerve compound action potential (CAP) and therefore the recorded CAP is of greater amplitude than the surface ABRs. However, such electrodes have the disadvantage that they are invasive and require a myringotomy for correct localisation, thus needing the expertise of an ear, nose, and throat surgeon, and have a placement time up to 45 minutes. ${ }^{11}$

To overcome these difficulties, we have adopted an alternative non-invasive method of monitoring auditory function using an extratympanic intrameatal electrode (IME) which records the CAP and is currently used in audiological practice to record ECoGs. ${ }^{14}$

In this paper we present our experience with intraoperative monitoring during excision of cerebellopontine angle tumours using an IME in parallel with ABRs recorded with surface electrodes. To our knowledge this represents 
Clinical and operative details of the monitored patients with preoperative and postoperative hearing results

\begin{tabular}{|c|c|c|c|c|c|c|c|c|}
\hline \multirow{3}{*}{$\begin{array}{l}\text { Patient } \\
\text { No }\end{array}$} & \multirow{3}{*}{$\begin{array}{l}\text { Tumour } \\
\text { type }\end{array}$} & \multirow{3}{*}{$\begin{array}{l}\text { Size } \\
(\mathrm{cm})\end{array}$} & \multirow{3}{*}{$\begin{array}{l}\text { Operative procedure } \\
\text { affecting waves }\end{array}$} & \multicolumn{4}{|c|}{ Audiogram } & \multirow{3}{*}{$\begin{array}{l}\text { Postoperative } \\
\text { Hearing }\end{array}$} \\
\hline & & & & \multicolumn{2}{|c|}{ Preoperative } & \multicolumn{2}{|c|}{ Postoperative } & \\
\hline & & & & $d B$ & $S D \%$ & $d B$ & $S D \%$ & \\
\hline 1 & AN & $<2.5$ & drilling & 70 & NA & 120 & NA & No \\
\hline 2 & CPA & $>2.5$ & drilling, tumour handling & NA & NA & NA & NA & No \\
\hline 3 & AN & $<2.5$ & drilling, tumour handling & 40 & NA & 95 & NA & Yes \\
\hline 4 & $\mathrm{AN}$ & $<2.5$ & drilling, tumour handling & 40 & NA & NA & NA & No \\
\hline 5 & AN & $>2.5$ & nerve section & 40 & NA & NA & NA & No \\
\hline 6 & AN & $<2.5$ & drilling & 50 & 100 & 55 & 60 & Yes \\
\hline 7 & AN & $>2.5$ & drilling, tumour handling & 20 & NA & NA & NA & No \\
\hline 8 & AN & $<2.5$ & Tumour handling & 50 & NA & NA & NA & No \\
\hline 9 & $\mathrm{CPA}$ & $>2.5$ & drilling & 30 & NA & 30 & NA & Yes* \\
\hline 10 & AN & $<2.5$ & diathermy, tmr handling & 30 & NA & NA & NA & No \\
\hline 11 & CPA & $>2.5$ & no major change & 20 & NA & 20 & NA & Yes* \\
\hline 12 & $\mathrm{CPA}$ & $>2.5$ & $\begin{array}{l}\text { Tumour handling, } \\
\text { hypotension }\end{array}$ & 10 & NA & 10 & NA & Yes $^{\star}$ \\
\hline 13 & AN & $<2.5$ & drilling, tumour enucleation & 30 & 100 & 30 & 95 & Yes* \\
\hline 14 & AN & $<2.5$ & Tumour handling & 20 & 90 & 20 & 90 & Yes* \\
\hline 15 & AN & $<2.5$ & nerve section & 20 & NA & - & - & No \\
\hline 16 & AN & $>2.5$ & no major change & 30 & NA & NA & NA & Yes \\
\hline 17 & AN & $<2.5$ & no major change & 55 & NA & 55 & NA & Yes \\
\hline 18 & AN & $<2.5$ & Vasospasm & 30 & 100 & 60 & NA & Yes \\
\hline 19 & AN & $<2.5$ & nerve section & 60 & 100 & - & - & No \\
\hline 20 & AN & $<2.5$ & Tumour handling & 45 & NA & 60 & NA & Yes \\
\hline 21 & AN & $<2.5$ & drilling & 30 & 100 & 40 & 100 & Yes $^{\star}$ \\
\hline 22 & AN & $<2.5$ & drilling & 30 & 100 & 40 & 100 & Yes* \\
\hline
\end{tabular}

$\mathrm{AN}=$ Acoustic neuroma $\mathrm{CPA}=$ cerebellopontine angle tumour; $\mathrm{NA}=$ not available $; \mathrm{dB}=$ hearing level in decibels at $1000 \mathrm{~Hz} ; \mathrm{SD}$ $=$ percentage of spoken words recognised; ${ }^{\star}$ Useful hearing.

the first systematic study of the use of an intrameatal extratympanic electrode to monitor the VIIIth nerve CAP during surgery for acoustic neuroma.

\section{Methods}

SUBJECTS

Twenty two patients with posterior fossa tumours, most of whom had acoustic neuromas and some degree of useful hearing, were considered for operative monitoring between May 1989 and March 1995. Twelve were men and 10 were women. Their ages ranged from 27 to 64 (mean 45.5) years. In 15 patients the tumour diameter was smaller than $2.5 \mathrm{~cm}$, and in seven patients it was greater than $2.5 \mathrm{~cm}$ (table). Tumour pathology confirmed acoustic neuromas in 18 patients $(15<2.5 \mathrm{~cm}, 3>2.5$ $\mathrm{cm}$ ) of whom one had neurofibromatosus II with bilateral acoustic neuromas. Four patients had other tumours of the cerebellopontine angle; two epidermoids and two meningiomas, all of which were more than $2.5 \mathrm{~cm}$ in diameter. Hearing loss was the major present-

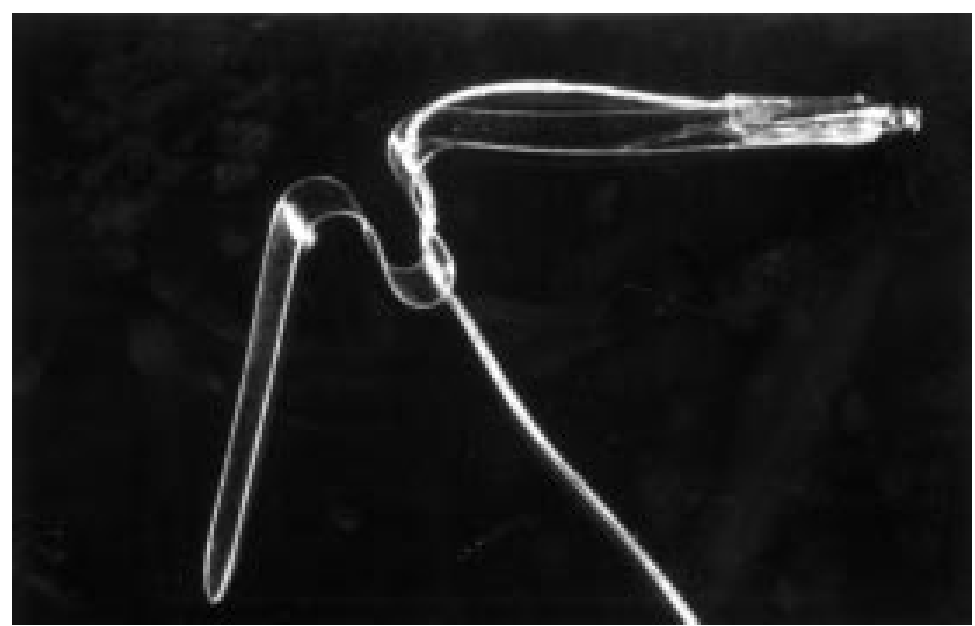

Figure 1 The intrameatal electrode. ing symptom in every case, but eight patients had additional symptoms of lower cranial nerve or brainstem dysfunction. Preoperative hearing status was assessed with pure tone audiograms in 21 patients, of whom 18 had useful hearing, defined for this study as being less than $50 \mathrm{~dB}$ loss at $1000 \mathrm{~Hz}$, and subjectively still able to discriminate speech. Three patients could only hear noise and were not able to discriminate speech in the affected ear. Two patients also had longstanding total deafness in the opposite ear.

\section{PROCEDURES}

Surgical procedure

All patients were operated on by the same neuro-otological surgeons (HBC and ARM) using the park bench position and retrosigmoid craniotomy. Anaesthesia was maintained with nitrous oxide, narcotic analgesia, and propofol infusion, and this combination least affected the evoked potential amplitude.

\section{Technical procedure}

Preoperatively all patients underwent baseline ABRs as well as pure tone audiograms, and the external meati were cleared of any wax. The meatal electrode (fig 1), its tip coated with electrode jelly, was inserted with ear forceps onto the posteroinferior quadrant of the tympanic membrane after induction of anaesthesia (fig 2). The lateral wings sprung open on insertion so that the electrode was held gently but firmly in place. The ABRs were also recorded using surface electrodes at the vertex and ipsilateral mastoid (Cz: active, mastoid:reference). Click stimuli at $11.1 \mathrm{~Hz} 97 \mathrm{~dB}$ sound pressure level were delivered by means of a Nicolet ear insert stimulating unit, with contralateral masking. The ear was sealed with bone wax and waterproof dressing. Recordings were undertaken with an 8400 Cadwell multichannel evoked potential machine with filters 


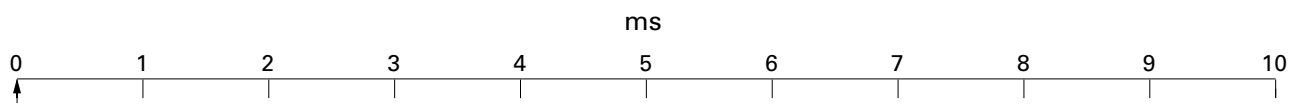

$\mathrm{ABR}$
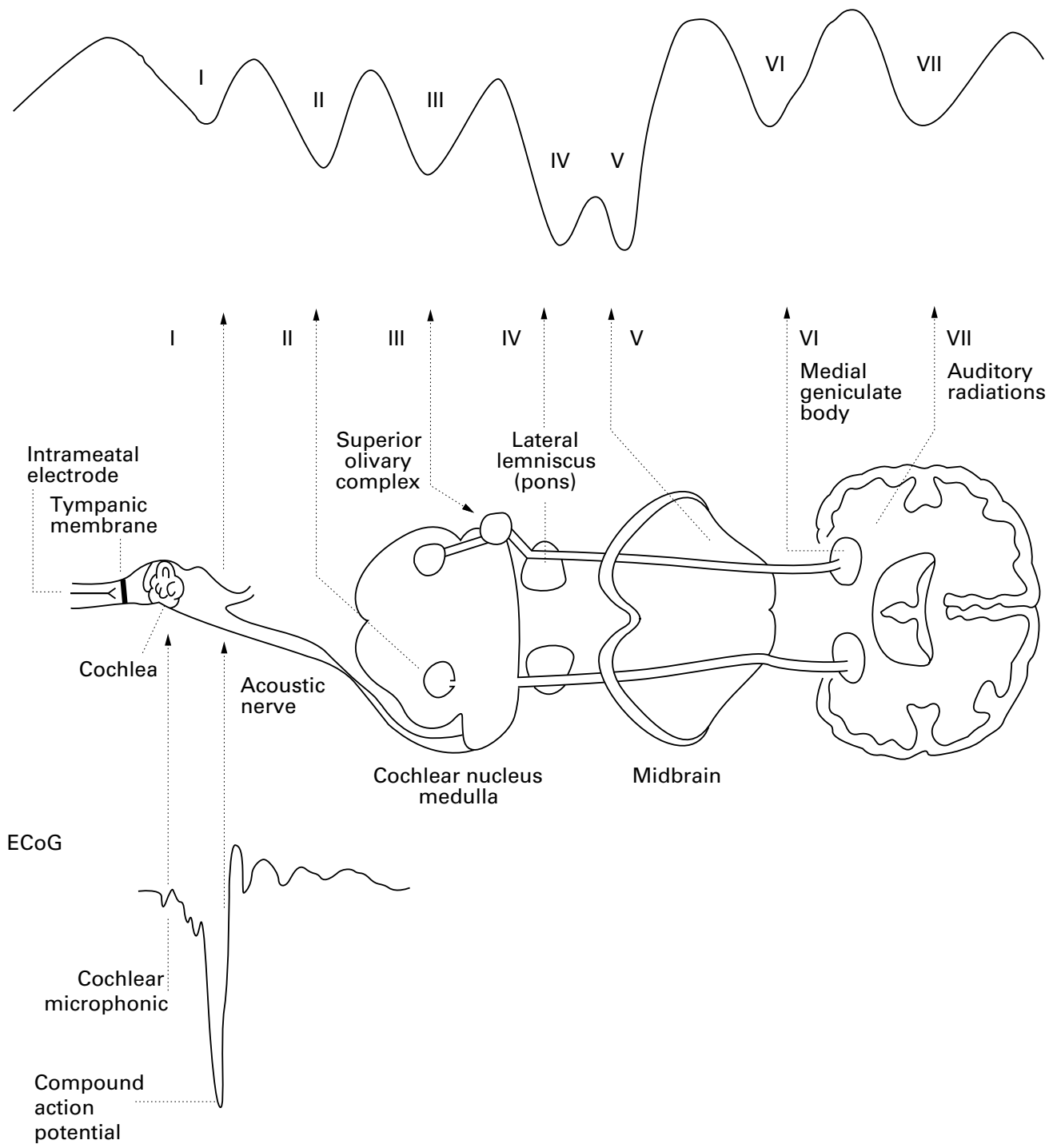

Figure 2 Presumed origins of the different components of the ECoG and ABR. The site of placement of the intrameatal electrode is shown.

between $30 \mathrm{~Hz}$ and $3 \mathrm{KHz}$ for both the ABRs and the ECoGs.

Figure 3 shows an example of the potentials recorded from the intrameatal electrode. The summation potential and the compound action potential (CAP) were both seen, but the latency and amplitude of the CAP as well as waveform morphology were monitored for any change. It is the changes in these latter results which are documented in this paper. Evoked potential data collection started as soon as the patient was prepared and was continuous throughout the operation. Baseline responses were recorded in the first 30 minutes of surgery and subsequently the surgeon was informed immediately of any variation from the steady baseline values which affected either the waveform amplitude, latency, or amplitude.

Simultaneous VIIth nerve monitoring was performed using intermittent stimulation, the facial muscle action potentials being recorded from the surface as well as subcutaneous electrodes in the orbicularis oculi, frontalis, orbicularis oris, and mentalis muscles using a Neuromatic 2000 EMG machine (Dantec).

\section{Results}

TECHNICAL RESULTS

All 22 patients who underwent intraoperative monitoring had discernible, although often relatively low amplitude, preoperative ABRs. The waves recorded by means of the IME were 


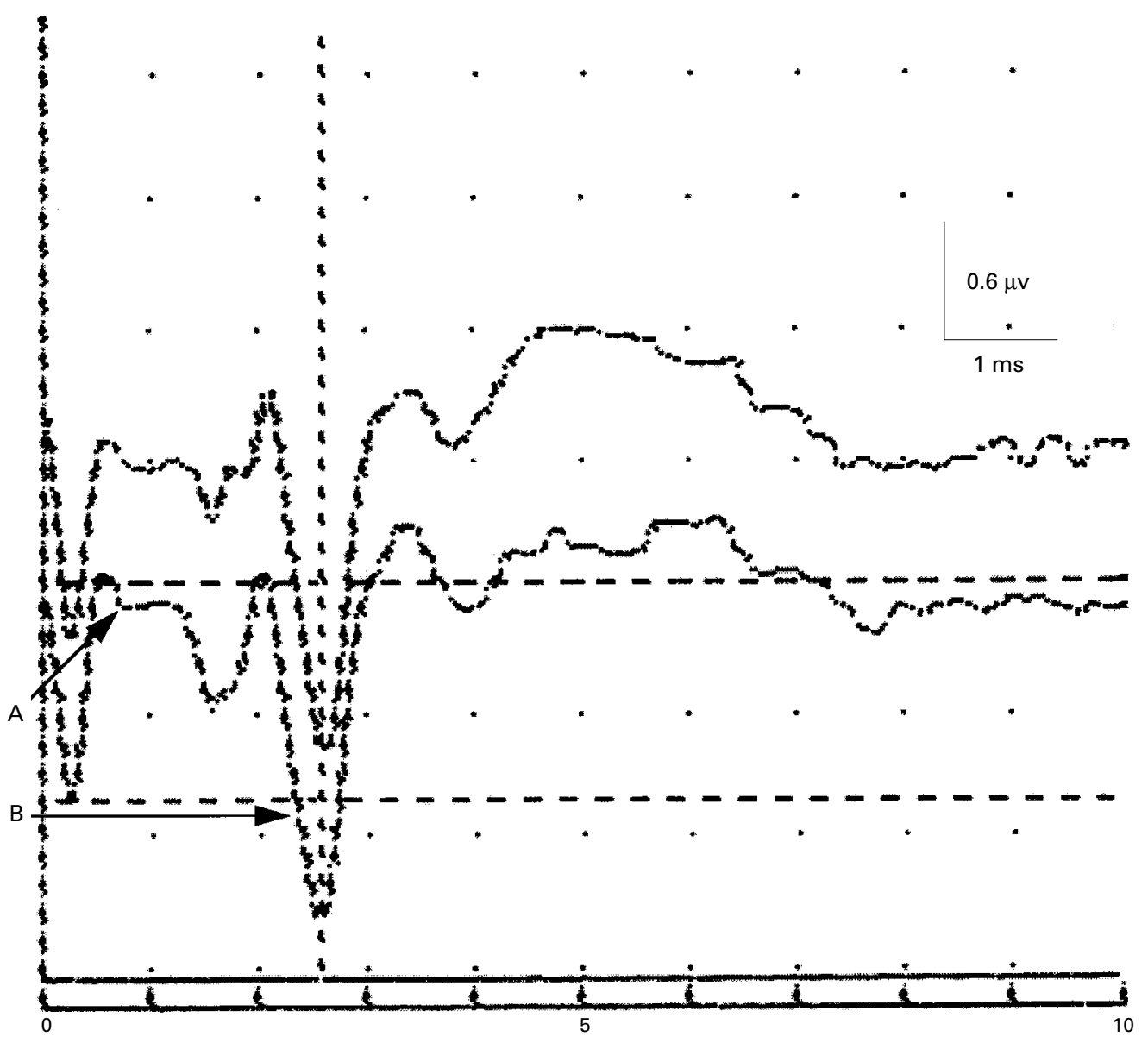

Figure 3 Components of the ECoG, seen as recorded with the IME in the operating theatre after anaesthesia was administered. Arrows show the summating potential (A) and compound action potential (B).

of relatively greater amplitude than those obtained with surface electrodes, the mean amplitude of wave I recorded by IME being about two and a half times greater than that recorded by surface electrodes (fig 4). Recorded potentials were retained throughout the operation in 13 patients (59\%), all of whom had preserved hearing after surgery. Three of these 13 patients had virtually unchanged stable responses throughout surgery. Nineteen patients developed significant temporary or permanent alteration of CAP amplitude and/or latency. Twenty patients showed significant transient amplitude or latency changes for wave V.

\section{OPERATIVE PROCEDURES AFFECTING MONITORED} POTENTIALS

Certain operative manoeuvres were particularly likely to be associated with changes in amplitude or latency of the monitored potentials.

Drilling around the internal auditory meatus Drilling of the posterior aspect of the temporal bone over the internal auditory meatus was a procedure which commonly caused prolongation of latency or drop in amplitude of the CAP as the earliest detectable change. This usually occurred in the first 15 to 30 minutes after commencing drilling. The operating surgeon would immediately be informed of any latency changes and he would then pause in the drilling and subsequently change from a cutting to a diamond burr. A pause of 5 to 10 minutes usually resulted in the reversal of the delayed latency, at which time drilling would be cautiously resumed with frequent pauses. When a pause did not improve latency or amplitude changes, the surgeon would then either make the decision to proceed cautiously with more frequent pauses during drilling, knowing that VIIIth nerve integrity was being threatened, or proceed, having decided that tumour removal took precedence over attempts at hearing preservation.

In 10 cases of acoustic neuroma drilling around the internal auditory meatus to expose the tumour caused notable changes in the latency and amplitude of the CAP (table). In one of these 10 patients (table, patient 1) the surgeons considered that they had no alternative but to continue drilling for full tumour exposure and the ECoG then progressively dropped in amplitude and disappeared. In this patient, with persistent loss of all waveforms, there was complete postoperative hearing loss. Of the other nine patients, the potentials reverted to normal in two (6 and 21) but some degree of abnormality persisted throughout the operation in the remaining seven patients. 


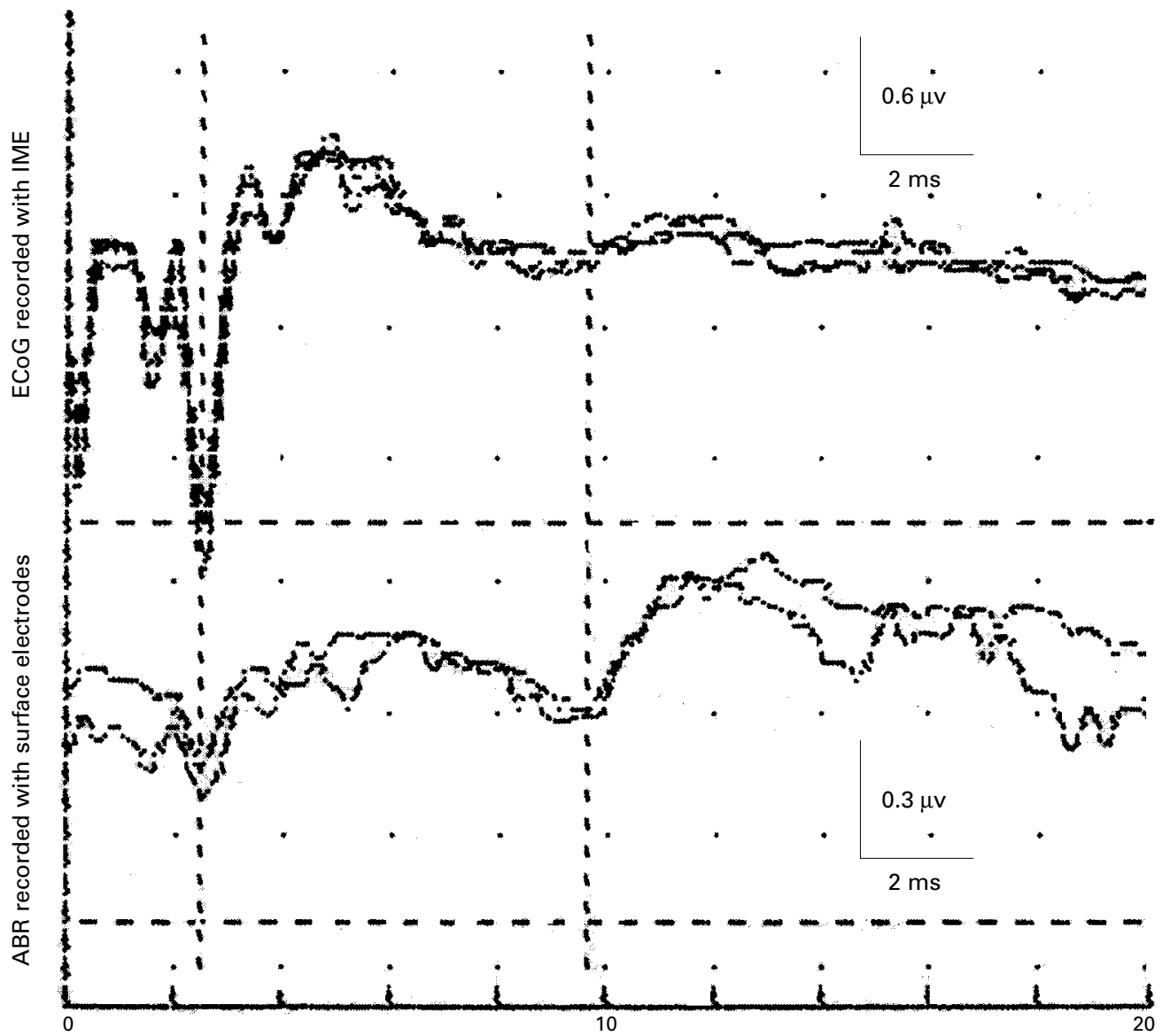

Figure 4 Recordings obtained at the start of surgery, after anaesthesia has been administered. The first cursor indicates the position of the CAP of the ECoG (upper trace) and wave I of the ABR (lower trace). The second cursor shows wave V. Note that the ECoG has been represented using lower sensitivity, to accommodate the CAP, hence the wave V is apparently small.

Operative procedures involved in tumour excision Operative manoeuvres such as nerve traction or tumour dissection caused a change in recorded potentials in 11 patients. This change reverted to normal in five patients but in six patients the CAP was irreversibly lost. All of these six patients had postoperative hearing loss. Retraction of the cerebellum, necessary to expose the tumour, caused transient wave $\mathrm{V}$ changes and never resulted in permanent hearing loss. Experience with the earlier patients led to changes in the surgical procedure, a decrease in the CAP causing the surgeon to pause and then to use an alternative operative approach if the CAP did not rapidly recover. Such measures were not always possible, and on some occasions removal of the tumour had to take priority over hearing preservation. In two instances (table, patients 5 and 19) the nerve was sectioned during tumour removal and in these patients the responses immediately disappeared.

In one patient with a $2 \mathrm{~cm}$ diameter acoustic neuroma tumour removal was being performed at a point distant from the VIIIth nerve when an acute and dramatic loss of potentials occurred, lasting a few minutes (table, patient 18). This event was therefore thought to be vascular in origin, possibly due to spasm of the cochlear artery, and local papaverine was applied. The CAP rapidly returned to about half its original amplitude, was then recorded throughout the operation, and the patient retained useful postoperative hearing. The CAP was lost totally in nine patients consequent to different surgical procedures (drilling one, nerve section two, nerve injury during tumour removal six). All of these patients had postoperative hearing loss.

RESULTS OF HEARING PRESERVATION

Hearing was preserved in 13 of the total 22 patients $(59 \%)$, and in 10 of 18 patients with acoustic neuromas (56\%) (table). Among those with acoustic neuromas, hearing was preserved more often when the tumour was smaller than $2.5 \mathrm{~cm}(9 / 15(60 \%))$ than when it was greater than $2.5 \mathrm{~cm}$ in diameter $(1 / 3$ $(33 \%))$. Among the four patients with other types of tumours of the cerebellopontine angle, hearing was preserved in three $(75 \%)$. Useful hearing was preserved in seven (33\%) patients. A further five patients await final assessment, and one patient had non-useful hearing.

The facial nerve was anatomically preserved in all patients. Immediately postoperatively 17 patients had normal facial muscle function (House grade 1), three had near normal function (House grade 2), and two had moderate facial palsy (House grade 3). 


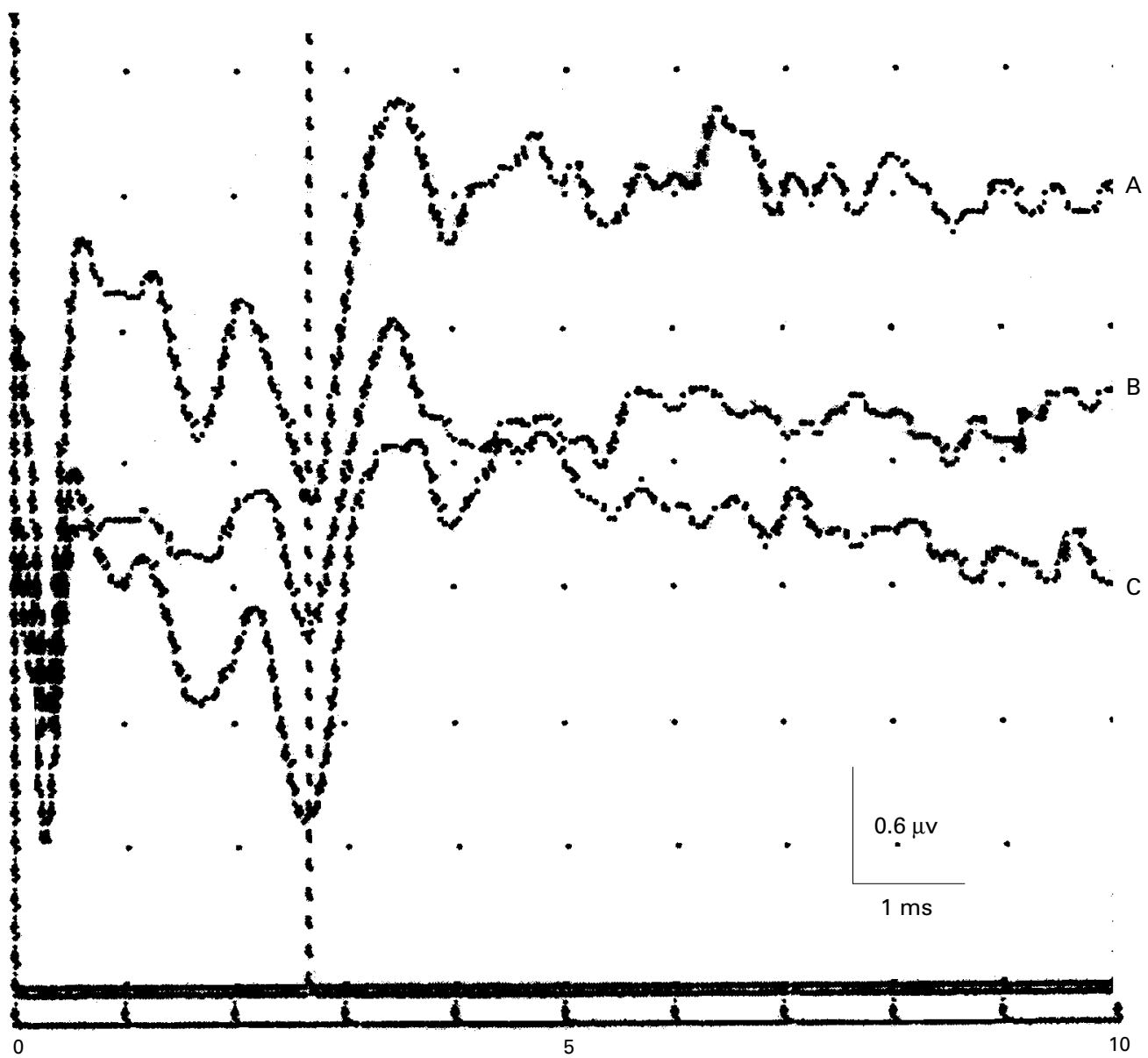

Figure 5 Consecutively recorded CAPs with the IME. The upper trace A, with CAP amplitude of $1.9 \mu \mathrm{V}$, was recorded at the start of drilling. This series was performed during the drilling of the internal auditory meatus (table 1, patient 21). The $C A P$ suddenly dropped in amplitude to $1.4 \mu \mathrm{V}(B)$. The surgeon immediately stopped drilling and waited. Over the next 5 minutes, the CAP amplitude gradually improved to $1.8 \mu \mathrm{V}(\mathrm{C})$. Drilling was then carefully resumed.

Discussion

The state of the art of intraoperative monitoring of the acoustic nerve, particularly during surgery for posterior fossa tumour, consists of near field recordings of the VIIIth nerve potential, either by transtympanic electrocochleography, ${ }^{12}{ }^{13}$ by direct recording of the VIIIth nerve potentials, ${ }^{9}$ or by recording from the lateral recess of the fourth ventricle. ${ }^{10}$ Recording with the extratympanic IME provided information which was on a par with that obtained by other means, and had minor disadvantages and particular benefits listed below.

\section{ADVANTAGES OF THE EXTRAYMPANIC ELECTRODE} Ease of placement

Information obtained by recording the ECoG using an extratympanic, intrameatal electrode and its most prominent component, the distally generated VIIIth nerve CAP, compares favourably with the information derived by the transtympanic electrode. Ruth and Lambert ${ }^{15}$ compared the use of (extra)tympanic ECoG using a foam tip electrode with a transtympanic ECoG derived using a needle electrode and advocated the first because of consistent waveform quality and ease of placement.

Although it is true that recording from the intrameatal electrode yields a lower amplitude
ECoG than that obtained with the transtympanic electrode, it is our experience that the CAP of the VIIIth nerve is sufficiently well defined for intraoperative monitoring to be a relatively simple and robust procedure. Roland et al compared simultaneous recordings from transtympanic and extratympanic electrodes in 19 healthy volunteers and found no significant difference in summating potential/action potential ratios used in evaluating inner ear fluid disorders. ${ }^{16}$ Winzenberg et $a l^{17}$ reviewed the use of extratympanic and transtympanic ECoG and also report that, in general, the transtympanic ECoG yielded a larger amplitude CAP. The major advantage noted with the extratympanic electrode was again its ease of application. These authors recommended supplementing the use of the ECoG with the ABR during intraoperative monitoring, as has been our practice.

Virtually instant information VIIIth nerve CAP We found that the extratympanic, intrameatal electrode yielded reliable and reproducible potentials which compared favourably with simultaneously recorded surface ABR responses. The CAP of the ECoG corresponded to the wave I of the ABR recorded with surface electrodes and the two covaried in amplitude and latency. The major benefit of recording 
with the IME was that the CAP in the ECoG was of greater amplitude than wave I of the $\mathrm{ABR}$, as the IME was nearer the generator site than the surface electrodes. This meant, therefore, that fewer responses needed to be averaged. Any changes in the waveforms during surgery could thus be detected in a matter of seconds, and sometimes even instantaneously, in the raw data.

\section{Non-invasive}

The IME had benefits over the transtympanic electrode in being non-invasive and therefore avoiding trauma to the tympanic membrane, a complication noted while monitoring with the transtympanic electrode. ${ }^{12}$ It was easily inserted by the neurosurgeon with the aid of the loupe, hence an ENT surgeon did not necessarily have to be present at the commencement of surgery.

\section{Permits continuous monitoring from beginning to} end of surgery

Recordings from the IME allowed continuous monitoring from the time the patient was anaesthetised until the end of surgery, an advantage over direct VIIIth nerve recording which can only be performed during the period of nerve exposure. It was only with continuous monitoring that the effects of drilling of the internal auditory meatus on VIIIth function became apparent.

Electrode placement distant from operative field The intrameatal electrode, by its location external to the tympanic membrane, was removed from the operative site and did not interfere with the operative field. Moller et al who first described monitoring with direct recording of the VIIIth nerve potential now agree that direct recording is an impediment in the operative field and recently advocated recording from the lateral recess of the fourth ventricle, ${ }^{10}$ to overcome that difficulty. However, the extratympanic IME does not have that disadvantage.

OPERATIVE PROCEDURES THAT AFFECTED VIIIth NERVE FUNCTION

Drilling of the internal auditory meatus

Monitoring with the intrameatal electrode disclosed some operative events that appeared to affect VIIIth nerve function. Drilling around the internal auditory meatus was an important cause of reduction in the CAP amplitude or change in latency of the CAP during surgery. Detection of this change in amplitude resulted in the surgeon pausing until the CAP recovered adequately before proceeding. It has become a matter of practice now for our surgeons to drill intermittently, with frequent pauses, to assess the integrity of the monitored potentials and allow irrigation of the drilling site with artificial CSF. It is not clear whether it is the heat generated by the drilling or the induction of high frequency vibrations which are responsible for the harmful effects. Changing the type of drill head to a diamond head seems to reduce the damage caused as this change was subsequently followed by a reduction in the degree of monitored change at this stage in the operation.

\section{Cerebellar retraction}

Cerebellar retraction at the onset of the procedure resulted in wave $\mathrm{V}$ changes. However, this never resulted in permanent damage to hearing, although retractor readjustment was occasionally necessary.

\section{Tumour handling}

Tumour handling, either movement of the whole tumour or dissection near the nerve, affected both the CAP and wave V. The surgeons have learned how to modify their dissection in terms of direction and gentleness. The clinical impression is that careful elevation of tumour tissue is safer than tumour movement in other directions.

Bipolar diathermy

This is now used only when essential.

\section{Vasospasm}

In one case (table, patient 18) the deterioration of the CAP and ABR was reversed with papaverine. Evoked otoacoustic emissions (EOAEs) have been reported to be useful, in conjunction with ABRs, to assess cochlear function during acoustic neuroma surgery and differentiate between (vascular) damage to the cochlea versus the VIIIth nerve system. ${ }^{18}$

A correlation between hearing preservation and preservation of the ECoG at the end of surgery has been shown in this series. Previous authors have noted that, on occasions, the CAP/ABR may persist after nerve section. ${ }^{712}$ As the CAP is thought to arise from the distal point of the VIIIth nerve, ${ }^{19}$ it is possible that injury to the nerve close to its origin from the brainstem may allow the CAP to be recorded for several days. In the present series, nerve section occurred mainly distally during tumour removal and the loss of CAP was postoperatively associated with hearing loss.

It has been our experience, therefore, that intraoperative monitoring has had prognostic implications for postoperative hearing. All patients with loss of CAP had postoperative hearing loss. Those patients with preserved CAP had preserved postoperative hearing albeit with a drop in PTA hearing thresholds or speech discrimination scores. Nadol et $a l^{20}{ }^{20}$ in their series of $78 \mathrm{ABR}$ monitored patients during acoustic neuroma excision, report preservation of wave $\mathrm{V}$ at the end of surgery was associated with preservation of hearing. Interestingly, loss of $\mathrm{N} 1$ of the CAP in 10 patients was invariably associated with postoperative hearing loss, which is similar to our own experience. Lenarz and Ernst, ${ }^{21}$ however, found postoperative hearing loss in only 12 of 16 patients who sustained a loss of N1 (or CAP) and wave V. It must be admitted, therefore, that a consensus is yet to emerge in literature on the prognostic implications of waveform loss.

Colletti and Fiorino ${ }^{22}$ reported their experience of monitoring the auditory nerve during surgery for acoustic neuroma in 14 patients. 
They found that drilling within the internal auditory canal, coagulation close to the auditory nerve and removal of the intrameatal portion of the tumour were the most critical steps for hearing loss during acoustic neuroma surgery.

In their patient group of 96 patients, Lenarz and Ernst ${ }^{21}$ report prolongation of $\mathrm{ABR}$ latencies and of N1 of the CAP as well as decrease of cochlear microphonic potential amplitudes in seven patients produced by intraoperative drilling. They found that ischaemia, either due to vasospasm or coagulation of the labyrinthine artery, resulted in loss of all potentials in seven of their 96 patients, prompt administration of a calcium antagonist such as nimodipine could reverse, in part, these changes. Dissection of the auditory nerve also caused changes in amplitude and latency of both the CAP of the ECoG and wave V, in 55 of their 96 patients.

In a recent report Matthies and $\mathrm{Samii}^{4}$ presented their experience of intraoperative monitoring in 200 patients with acoustic neuromas, using auditory brainstem responses, and studied the effect of various operative manoeuvres on waves I, III, and V and the correlative changes with hearing loss. They report wave III loss, followed by wave V and wave I loss, as the most sensitive indicators for temporary or long term deafness. The surgical manipulations noted to be most harmful were drilling, pulling downward, medially or laterally, and direct nerve manipulation. They conclude that useful and timely recognition of significant waveforms is possible, enabling a change of microsurgical manoeuvres which favour $A B R$ recovery. It has been our experience, however, with simultaneous monitoring of the CAP using the intrameatal electrode and the $A B R$, that the first gave much quicker information, being practically instantaneous. There was no delay required for averaging and therefore the surgeon was informed much sooner. In addition, the CAP was usually of a reasonable amplitude, being recorded as a near field potential as opposed to far field $A B R$ recordings, and this enabled easy identification of changes in waveform amplitude or morphology. We noted that, particularly in hearing impaired patients, identifying waves I and III of the $A B R$ was difficult, though wave $V$ was often obvious. Monitoring of the ABR inside the operating theatre was affected by additional technical difficulties, as the anaesthesia itself was liable to cause a further drop in amplitude. Although we monitored both the CAP and the ABRs, it was the CAP which proved to be the more useful, reliable, and easy to interpret. Gianotta $^{23}$ has echoed the opinions regarding the technical difficulties of monitoring the ABR in a follow on comment to the paper by Matthies and Samiis.

We have a strong clinical impression that hearing preservation has been facilitated by this technique of intraoperative monitoring. In a previous report from this centre, with the same group of surgeons, hearing preservation was possible in about $30 \%$ of all acoustic neuromas irrespective of size ${ }^{1}$. It is generally accepted that hearing preservation has the best prognosis when the tumour is less than $2 \mathrm{~cm}$ in size and there is good postoperative hearing. ${ }^{22-26}$ In this series, after intraoperative monitoring had been instituted, hearing preservation in tumours smaller than $2.5 \mathrm{~cm}$ improved to $60 \%$ (9/16), although in those larger than $2.5 \mathrm{~cm}$ it remained at $33 \%(1 / 3)$. The reasons for this must include other factors, but this aspect of the procedure warrants further assessment by other centres to confirm this encouraging finding.

Finally, direct recording of the compound action potential with a surgical probe, the acoustic nerve detector (AND) placed on the cochlear fibres is also a useful aid to hearing preservation, particularly as the cochlear fibres can be anatomically identified during surgical dissection. ${ }^{27}$ This instrument is being further developed within this group (SRB). ${ }^{27}$

In conclusion, the use of monitoring allows instant identification of auditory nerve changes and the possibility of action to limit the damage sustained. In this series the use of monitoring disclosed that inadvertent and unrecognised loss of hearing could occur at the time of drilling early in the operation. This is a preventable occurrence. The modification of surgical technique during tumour removal is more controversial, as it could be argued that tumour removal might then be less rigorous. However, on balance it is our view that the benefits probably outweigh the disadvantages and the method described is one which is relatively simple to apply in all neurosurgical centres.

1 Torrens M, Maw R, Coakham H, et al. Facial and acoustic nerve preservation during excision of extracanalicular acoustic neuromas using the suboccipital approach. $B r F$ Neurosurg 1994;8:655-65.

2 Samii M, Matthies C. Management of 1000 vestibular schwannomas (acoustic neuromas): hearing function in 1000 tumour resections. Neurosurgery 1997;40:248.

3 Symon L, Bordi LT, Compton JS, et al. Acoustic neuroma: a review of 392 cases. Br 7 Neurosurg 1989;3:343-8.

4 Matthies C, Samii M. Management of vestibular schwannomas (acoustic neuromas): the value of neurophysiology for intraoperative monitoring of auditory function in 200 intraoperative monitoring of auditor

5 Harper CM, Harner SG, Slavit DH, et al. Effect of BAEP monitoring on hearing preservation during acoustic monitoring on hearing preservation during

6 Friedman WA, Kaplan BJ, Gravenstein D, et al. Intraoperative brain-stem auditory potentials during posterior fossa microvascular decompression. $\mathcal{F}$ Neurosurg 1985;62:552-7.

7 Raudzens PA, Shelton AG. Intraoperative monitoring of brain-stem auditory evoked potentials. F Neurosurg 1982; 57:341-8.

8 Grundy BL, Jannetta PJ, Procopio PT, et al. Intraoperative monitoring of brain-stem auditory evoked potentials. $\mathcal{F}$ Neurosurg 1982;57:674-81.

9 Moller AR, Jannetta J. Monitoring auditory functions during cranial nerve microvascular decompressions by direct recording from the eighth nerve. $\mathcal{f}$ Neurosurg 1983;59:493-9.

10 Moller AR, Jho HD, Jannetta PJ. Preservation of hearing in operations on acoustic tumours: an alternative to recording operations on acoustic tumours: an alternative to recording
brainstem auditory potentials. Neurosurgery 1994;34:68893.

11 Albuquerque FC, Gianotta SL. Cranial nerve preservation in surgery of the cerebellopontine angle. Neurosurgery Quarterly 1996;6:31-50

12 Symon L, Sabin HI, Bentivoglio P, et al. Intraoperative monitoring of the electrocochleogram and preservation of hearing during acoustic neuroma excision. Acta Neurochir (Wein) 1988; (suppl 42): 27-30.

13 Ojemann RG, Levine RA, Montgomery WM, et al. Use of intraoperative auditory evoked potentials to preserve hearing in unilateral acoustic neuroma removal. $\mathcal{F}$ Neurosurg 1984;61:938-48.

14 Coats AC. On electrocochleographic electrode design. $\mathcal{f}$ Acoust Soc Am 1974;56:708-11.

15 Ruth RA, Lambert PR. Comparison of tympanic membrane to promontory electrode recordings of electrocochleoto promontory electrode recordings of electrocochleo-
graphic responses in patients with Menieres disease. graphic responses in patients with Menier
Otolaryngol Head Neck Surg 1989;100:546-55.

16 Roland PS, Yellin MW, Meyerhoff WL, et al. Simultaneous comparison between transtympanic and extratympanic electrocochleography. Am f Otol 1995;16:444-50. 
17 Winzenberg SM, Margolis RH, Levine SC, et al. Tympanic and transtympanic electrocochleography in acoustic neuroma and vestibular nerve section surgery. Am f Otol 1993

18 Cane MA, O'Donoghue GM, Lutman ME. The feasibility of using oto-acoustic emissions to monitor cochlear function during acoustic neuroma surgery. Scand Audiol 1992;21:173-76

19 Moller AR. Auditory neurophysiology. F Clin Neurophysiol 1994;11:284-308.

20 Nadol JB, Levine R, Martuza RL, et al. Preservation of hearing in surgical removal of acoustic neuromas of the internal auditory canal and cerebellopontine angle. Laryngoscope 1987;974:1287-94.

21 Lenarz T, Ernst A. Intraoperative monitoring by transtympanic electrocochleography and brainstem electric response audiometry in acoustic neuroma surgery. Eur Arch Otorhinolaryngol 1992;249:257-62.
22 Colletti V, Bricolo A, Fiorino FG, et al. Changes in directly recorded cochlear nerve compound action potentials during acoustic tumour surgery. Skull Base Surgery 1994;4: $1-9$.

23 Gianotta SL. Comments. Neurosurgery 1997;40:466.

24 Umezu H, Aiba T. Preservation of hearing after surgery for acoustic schwannomas: correlation between cochlear nerve function and operative findings. F Neurosurg 1994;80:8448.

25 Sanna M, Karmarker S. Landolfi M. Hearing preservation in vestibular schwannoma surgery: fact or fantasy? F Laryngol Otol 1995;109:374-80.

26 Baldwin DL, King TT, Morrison AW. Hearing conservation in acoustic neuroma surgery via the posterior fossa. $\mathcal{F}$ Laryngol Otol 1990;104:463-7.

27 Butler S, Coakham H, Maw R, Morgan H. Physiological identification of the auditory nerve during surgery for identification of the auditory nerve during surger
acoustic neuroma. Clin Otolaryngol 1995;20:312-17. 